\title{
High Expression of Tomm34 and Its Correlations With Clinicopathology in Oral Squamous Cell Carcinoma
}

Min Cai, Rukeng Tan, Yunyi Huang, Xuanyi Chen, Qingci Kong, Kaixin Guo and Meng Xu*

Hospital of Stomatology, Guanghua School of Stomatology, Sun Yat-sen University, Guangdong Provincial Key Laboratory of Stomatology, Guangzhou, China

Tomm34, as a member of the outer mitochondrial membrane proteins, is evenly distributed between the cytoplasm and the outer mitochondrial membrane. It is up-regulated in a variety of tumors and correlates with poor prognosis. This study aimed to investigate expression of Tomm34 and its correlations with clinicopathology in oral squamous cell carcinoma (OSCC). Oncomine database and UALCAN database were utilized to predict the expression and prognosis values of Tomm34 in head and neck squamous cell carcinoma (HNSCC). By immunohistochemistry, a retrospective study was performed to verify the bioinformatics results to evaluate the Tomm34 expression and clinicopathological variables in both HPVpositive OSCC and HPV-negative OSCC. Immunohistochemistry of our cohort revealed that 48 cases fulfilled the Tomm34 high expression judgment criteria, and the overall positive rate was 60\% (48/80), and 27 cases fulfilled the p16 expression judgment criteria (33.75\%, 27/ 80). The high expression of Tomm34 was closely related with the TNM classification of OSCC $(p<0.01)$ and tumor size $(p<0.01)$ both in HPV-negative OSCC and HPV-positive OSCC, while related with lymph node metastasis $(p=0.001)$ in HPV-negative OSCC and drinking history $(p=0.044)$ in HPV-positive OSCC. In addition, the Kaplan-Meier curves indicated that higher level of Tomm34 was correlated with poorer overall survival (OS) and disease-free survival (DFS) in HPV-negative OSCC (OS, $p=0.046$; DFS, $p=0.020$ ) but not in

OPEN ACCESS

Edited by: Gabor Cserni, University of Szeged, Hungary

*Correspondence: Meng $X_{U}$ xumeng23@mail.sysu.edu.cn

Received: 13 December 2020 Accepted: 02 March 2021 Published: 16 April 2021

Citation:

Cai M, Tan R, Huang Y, Chen X, Kong Q, Guo K and Xu M (2021) High Expression of Tomm34 and lts Correlations With Clinicopathology in Oral Squamous Cell Carcinoma. Pathol. Oncol. Res. 27:641042. doi: 10.3389/pore.2021.641042 HPV-positive OSCC (OS, $p=0.824$; DFS, $p=0.782$ ). In conclusion, Tomm34 is highly expressed in OSCC and may be a useful factor to provide prognostic information, especially in HPV-negative OSCC group.

Keywords: oral squamous cell carcinoma, Tomm34, Immunohistochemistry, clinicopathology, bioinformatics

\section{INTRODUCTION}

Head and neck squamous cell carcinoma (HNSCC), accounts for approximately $80 \%$ of head and neck tumors, is highly aggressive with frequent local recurrences and lymph node metastases [1]. Oral cavity, oropharynx, larynx are the most common sites of HNSCC [2]. The routine therapy for oral squamous cell carcinoma (OSCC) patients is radical surgical resection combined with chemotherapy and radiotherapy, whereas the outcome is unsatisfactory. About half of patients with advanced cancer die within 5 years [3,4]. Early diagnosis of OSCC is important in patient treatment and prognostic evaluation. Therefore, it is worthwhile to reveal novel diagnostic and prognostic biomarkers for OSCC.

Recently, the role of high-risk human papilloma virus (HPV) infection in HNSCC has been wellconfirmed [5]. The HPV-associated oropharyngeal carcinoma (HPV-OPC) has been considered as a 
A

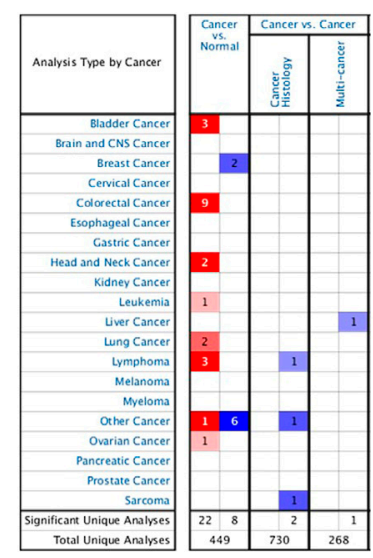

C

Expression of TOMM34 in HNSC based on Sample types ${ }_{80}{ }^{P}<4,20-14$

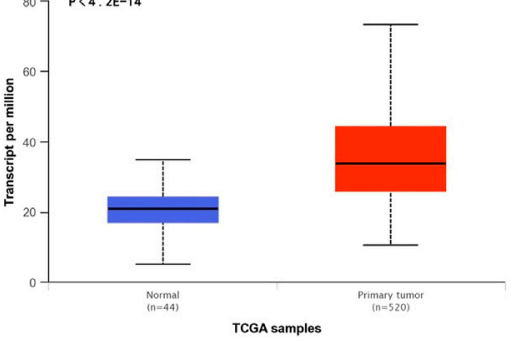

E

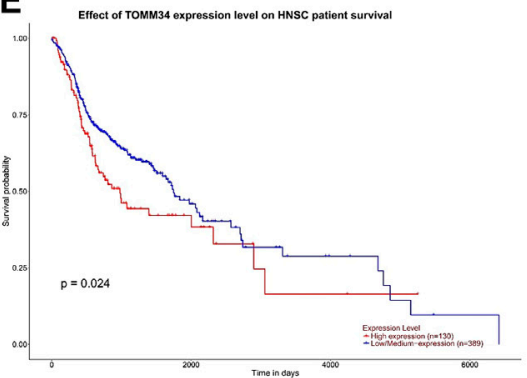

B

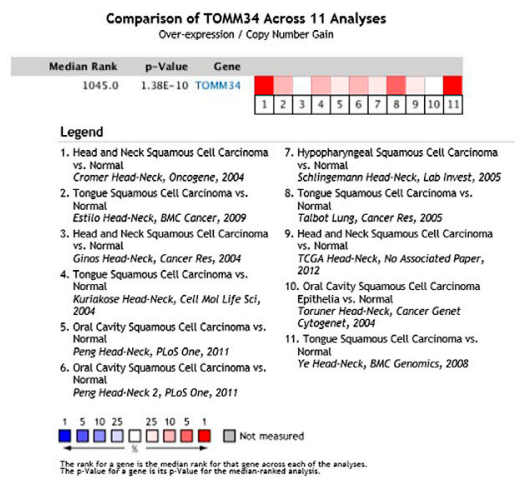

D

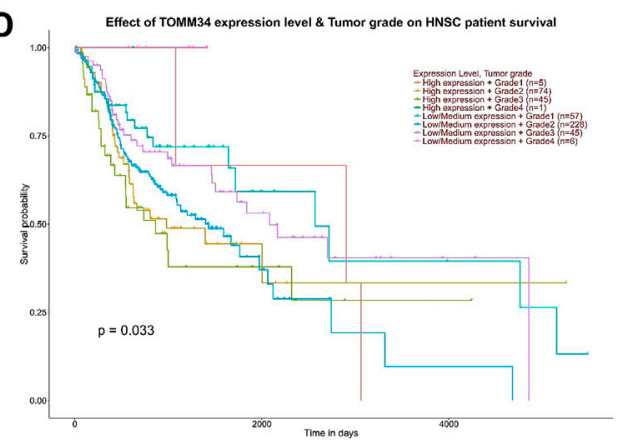

FIGURE 1 | (A) Tomm34 expression levels in human cancer tissues. (B) Comparison of Tomm34 across 11 analyses in HNSCC. (C) Expression of Tomm34 based on HNSCC sample types. (D) Tomm34 expression varies in HNSCC with different grades (The categories of tumor grade: Grade1, well differentiated; Grade2, moderately differentiated; Grade3, poorly differentiated; Grade4, undifferentiated [22]). (E) Kaplan-Meier survival tests comparing in the high and low/medium expression of Tomm34 in HNSCC.

distinct entity according to the WHO classification ( $4^{\text {th }}$ edition) [6-8], in addition, OSCC has been validated to harbor the virus as well. It is widely demonstrated that p16 immunohistochemistry can be utilized as a surrogate marker to classify the HPV status in OPC and OSCC [5, 9]. HPV-positive OPC exhibited a more favorable prognosis than HPV-negative OPC, however, the mechanism of better prognosis of HPV-positive OSCC compared with HPV-negative OSCC has not been fully understood yet.

Tomm34 (34-kDa translocase of the outer mitochondrial membrane), a member of the TOMM family, locates on chromosome 20q13.12 and contains 18,357 bases and 6 TPR repeats $[10,11]$. It is evenly distributed between the cytoplasm and the outer mitochondrial membrane and participates in protein transport [12]. Tomm34 can interact with Hsp70 and
Hsp90 to form Hsp70-Tomm34-Hsp90 complex, which acts as a co-chaperon of Hsp70 and Hsp90 to regulate its function [13, 14]. Recent studies showed high levels of Tomm34 expressed in colorectal cancer [15], hepatocellular carcinoma [16], bladder cancer [17], breast cancer [18, 19] epithelial ovarian cancer [20] and colon cancer [21] compared to their normal tissue counterparts. However, the expression of Tomm34 in oral squamous cell carcinoma primary tumor has not been reported and its role in OSCC is still unclear.

In the present study, we aimed to investigate the expression of Tomm34 in OSCC. Oncomine database and UALCAN database were utilized to predict the expression and prognosis values of Tomm34 in HNSCC. By immunohistochemistry, a retrospective study was performed to verify the bioinformatics results to evaluate Tomm34 expression and clinicopathological variables. 


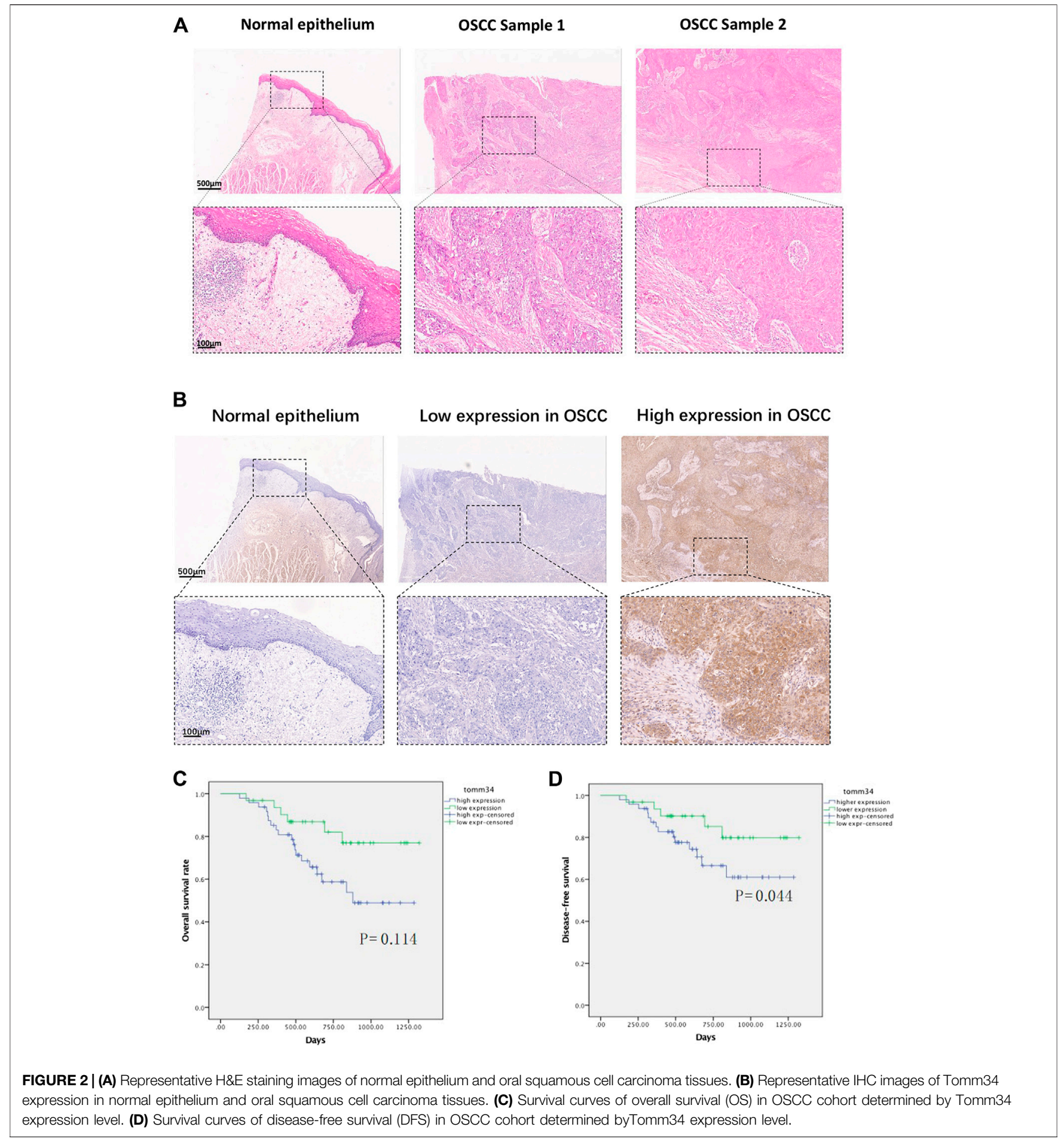

\section{MATERIALS AND METHODS}

\section{Tomm34 Expression in HNSCC Analyzed by}

\section{Oncomine Database}

Oncomine is an online data-mining platform which contains various microarray expression data. The data of Tomm34 gene expression in HNSCC was extracted from Oncomine database. The search conditions were as follows: 1) Gene: Tomm34; 2) Analysis Type: Cancer vs. Normal Analysis; 3) Cancer Type: Head and Neck Squamous Cell Carcinoma; 4) Analysis parameters were $p$-value $<1 \mathrm{E}-4$, fold change $>2$, gene rank $=10 \%$. 
TABLE 1 | Association between $\mathrm{IHC}$ expression of Tomm34 and clinicopathological parameters in the studied cohort $(n=80)$.

\begin{tabular}{|c|c|c|c|c|}
\hline & \multicolumn{2}{|c|}{ Tomm34 expression } & \multicolumn{2}{|c|}{ Significance } \\
\hline & Negative & Positive & $x^{2}$ & $p$ Value \\
\hline \multicolumn{5}{|l|}{ Age } \\
\hline$\leq 60$ (years) & 24 & 33 & 0.366 & 0.545 \\
\hline$>60$ (years) & 8 & 15 & & \\
\hline \multicolumn{5}{|l|}{ Gender } \\
\hline Male & 23 & 32 & 0.242 & 0.622 \\
\hline Female & 9 & 16 & & \\
\hline \multicolumn{5}{|l|}{ Tumor size } \\
\hline $\mathrm{T} 1 / \mathrm{T} 2$ & 30 & 24 & 16.752 & $<0.001$ \\
\hline $\mathrm{T} 3 / \mathrm{T} 4$ & 2 & 24 & & \\
\hline \multicolumn{5}{|l|}{ Node category } \\
\hline $\mathrm{N}^{-}$ & 27 & 23 & 0.668 & 0.001 \\
\hline $\mathrm{N}^{+}$ & 5 & 25 & & \\
\hline \multicolumn{5}{|l|}{ Pathology differentiation } \\
\hline Well & 14 & 21 & 0.000 & 1.000 \\
\hline Moderate/poor & 18 & 27 & & \\
\hline \multicolumn{5}{|l|}{ TNM classification } \\
\hline I/II & 25 & 10 & 25.608 & $<0.001$ \\
\hline III/IV & 7 & 38 & & \\
\hline \multicolumn{5}{|l|}{ Smoking } \\
\hline Yes & 8 & 18 & 1.368 & 0.242 \\
\hline No & 24 & 30 & & \\
\hline \multicolumn{5}{|l|}{ Alcohol (>5g/Day) } \\
\hline Yes & 2 & 13 & 5.470 & 0.019 \\
\hline No & 30 & 35 & & \\
\hline \multicolumn{5}{|l|}{ Site } \\
\hline Buccal mucosa & 2 & 9 & 4.705 & 0.453 \\
\hline Floor of mouth & 3 & 6 & & \\
\hline Gingiva & 3 & 6 & & \\
\hline Oropharynx and soft palate & 2 & 2 & & \\
\hline Hard palate & 1 & 3 & & \\
\hline Tongue & 21 & 22 & & \\
\hline
\end{tabular}

\section{Kaplan-Meier Survival Analysis of the Tomm34 Analyzed by UALCAN}

UALCAN is a comprehensive, user-friendly, and interactive web resource for analyzing cancer OMICS data [22]. Transcripts per million (TPM) values were used to measure the expression level of Tomm34, categorized into high expression group (TPM values are above the upper quartile) and low/medium expression group (TPM values are below the upper quartile) [22]. We compared the expression of Tomm34 in normal tissues and HNSCC, and analyzed the correlation of Tomm34 with clinicopathological variables such as tumor stage, tumor classification, race and weight and other indicators. Kaplan-Meier curves of Tomm34 expression and survival time were also evaluated.

\section{Primary OSCC Samples}

80 archival formalin-fixed paraffin embedded (FFPE) primary OSCC biopsies were recruited from the tissue bank of Department of Oral Pathology, Hospital of Stomatology, Guanghua School of Stomatology, Sun Yat-sen University, Guangzhou, China for immunohistochemical (IHC) staining study. All of the specimens were histologically evaluated as squamous cell carcinoma which originated from oral cavity and oropharynx including tongue, buccal mucosa, gingiva, floor of mouth, hard palate, oropharynx and soft palate. Clinicopathological informations including gender, age, tumor size, lymph node metastasis, pathology differentiation, TNM status, disease-free survival (DFS) and overall survival (OS) were analyzed. The study protocol was approved by Clinical Research Ethics Committee of Hospital of Stomatology, Guanghua School of Stomatology, Sun Yat-sen University.

\section{Immunohistochemistry}

FFPE OSCC specimens were cut into $4 \mu \mathrm{m}$ thick sections, deparaffinized in xylene, and rehydrated in serial dilutions of ethanol. Endogenous peroxidase activities were subsequently blocked by a 10 -min treatment with $3 \% \mathrm{H}_{2} \mathrm{O}_{2}$ solution. Antigen retrieval was performed in EDTA ( $\mathrm{pH}$ 9.0) for $2 \mathrm{~min}$ at $10.0 \mathrm{MPa}$. Following incubation in $5 \%$ BSA for $10 \mathrm{~min}$ to block endogenous biotin activity, sections were incubated with the primary antibody (Tomm34, 1:100, Proteintech Inc., United States; p16, 1:200, Abcam, United States) at $4^{\circ} \mathrm{C}$ overnight. The HRP-labeled secondary antibody was applied to the sections and incubated at $37^{\circ} \mathrm{C}$ for $40 \mathrm{~min}$. For color development, sections were stained $3,3^{\prime}$ diamino-benzidine (DAB) substrate and hematoxylin. Human colon carcinoma tissue was utilized as the positive control, and samples which were incubated with PBS instead of primary antibody served as the negative controls.

The extent and intensity of staining were evaluated and scored by two independent pathologists. Twenty microscopic high-power fields of tumor tissues were randomly selected for assessment. A numeric intensity score of 0-4 was assigned to each case based on the percentage of positive neoplasm cells $(0$, negative, $<10 \%$ positive cells staining; $1+, 10-25 \%$ positive cells with weak staining; $2+$, $25-50 \%$ positive cells with moderate staining; $3+, 51-70 \%$ positive cells with strong staining and $4+,>70 \%$ positive cells with strong staining). Staining was dichotomized into low expression and high expression by score. Score $0-2$ was considered as low expression of Tomm34, and score 3-4 was considered as high expression of Tomm34. Meanwhile, $>70 \%$ positive cells with strong and diffuse nuclei staining (with or without cytoplasmic staining) (score 4) was considered as positive expression of p16 [9].

\section{Statistical Analyses}

Data was analyzed by IBM SPSS statistical software V22 (IBM Corporation, United States). The $\chi 2$ test was utilized to assess the associations between the expression of Tomm34 or p16 and clinical characteristics of patients. Survival estimates were analyzed by Kaplan-Meier method with significance determined by the log rank test. A $p$ value less than 0.05 (twosided) was considered significant.

\section{RESULTS}

\section{Tomm34 Expression in Different Tumors by Oncomine Database}

A total of 449 cases of different tumors of Tomm34 gene research results were collected in the Oncomine database. 30 research 


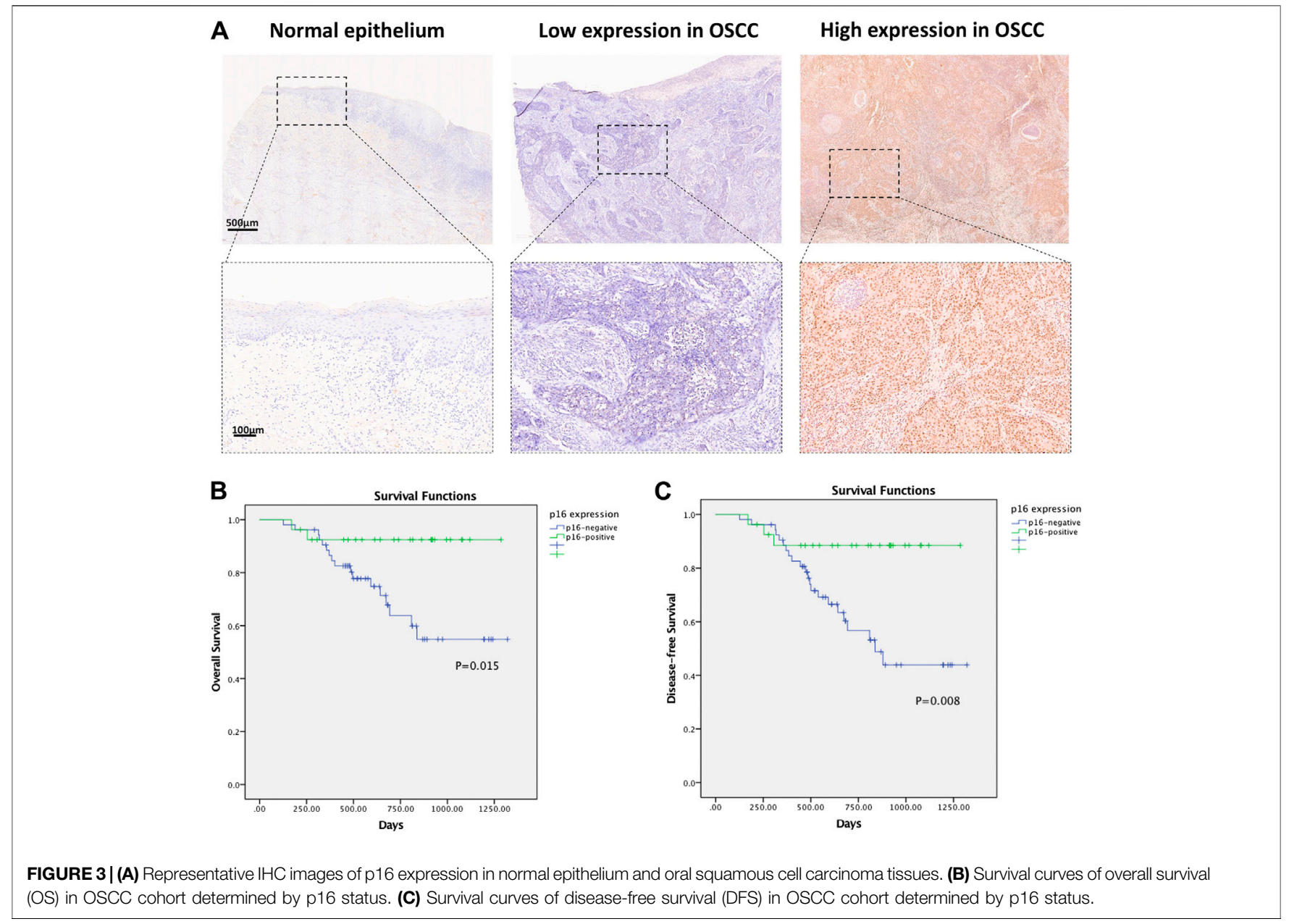

results showed statistical differences, where 22 of 30 cases reported overexpression of Tomm34 in tumor tissues and 8 cases reported lower expression in tumor tissues (Figure 1A).

\section{Tomm34 Expression in HNSCC by Oncomine Database}

Tomm34 gene ranked in 1,045 position among the total expressed genes of $\operatorname{HNSCC}(p=1.38 \mathrm{E}-10)$ (Figure 1B). Moreover, Tomm34 expression was significantly upregulated in the HNSCC tissues paired normal tissues (Figure 1C).

\section{Tomm34 Expression and Clinicopathological Correlations in HNSCC Analyzed via UALCAN}

For further study, the expression of Tomm34 in HNSCC was investigated in subgroups via UALCAN analysis. Tomm34 expression varied in HNSCC with different grades (Figure 1D). The Kaplan-Meier curves showed that higher level of Tomm34 correlated with poorer overall survival (OS) (Figure 1E).

\section{Tomm34 Expression and Its Correlations With Clinicopathology in Primary OSCC}

Tomm34 even staining was seen in the cytoplasm of tumor cells, nuclear expression was rarely seen (Figures 2A,B). Weak positive expression was seen in keratinocytes and some tumor stroma. In the cohort of 80 OSCC, 48 cases fulfilled the Tomm 34 positive judgment criteria, and the overall positive rate was $60 \%$.

The relationship between Tomm34 staining and clinicopathological factors were shown in Table 1. The expression of Tomm34 was closely related with the TNM clinical stage of OSCC $(p<0.001)$, tumor size $(p<0.001)$, lymph node metastasis $(p=0.001)$, drinking history $(p=0.019)$, but not with pathology differentiation, age, gender, smoking history and site $(p>0.05)$.

To investigate the effects of Tomm 34 on patient survival in more detail, we next assessed the relationship between the Tomm34 expression level and patient overall survival by Kaplan-Meier survival analysis. The overall survival was extremely down-regulated in the Tomm34-positive expression group compared with the Tomm34-negative expression group. The Kaplan-Meier curves indicated that higher level of Tomm34 correlated with poorer overall 
TABLE 2 | Association between IHC expression of p16 and clinicopathological parameters in the studied cohort $(n=80)$.

\begin{tabular}{|c|c|c|c|c|}
\hline & \multicolumn{2}{|c|}{ p16 expression } & \multicolumn{2}{|c|}{ Significance } \\
\hline & Negative & Positive & $x^{2}$ & $p$ Value \\
\hline \multicolumn{5}{|l|}{ Age } \\
\hline$\leq 60$ (years) & 36 & 21 & 0.848 & 0.357 \\
\hline > 60 (years) & 17 & 6 & & \\
\hline \multicolumn{5}{|l|}{ Gender } \\
\hline Male & 37 & 18 & 0.082 & 0.774 \\
\hline Female & 16 & 9 & & \\
\hline \multicolumn{5}{|l|}{ Tumor size } \\
\hline $\mathrm{T} 1 / \mathrm{T} 2$ & 35 & 19 & 0.153 & 0.696 \\
\hline T3/T4 & 18 & 8 & & \\
\hline \multicolumn{5}{|l|}{ Node category } \\
\hline $\mathrm{N}^{-}$ & 27 & 23 & 8.949 & 0.003 \\
\hline $\mathrm{N}^{+}$ & 26 & 4 & & \\
\hline \multicolumn{5}{|l|}{ Pathology differentiation } \\
\hline Well & 24 & 11 & 0.150 & 0.699 \\
\hline Moderate/poor & 29 & 16 & & \\
\hline \multicolumn{5}{|l|}{ TNM classification } \\
\hline$|/| \mid$ & 19 & 16 & 3.983 & 0.046 \\
\hline III/IV & 34 & 11 & & \\
\hline \multicolumn{5}{|l|}{ Smoking } \\
\hline Yes & 14 & 12 & 2.650 & 0.104 \\
\hline No & 39 & 15 & & \\
\hline \multicolumn{5}{|l|}{ Alcohol (>5g/Day) } \\
\hline Yes & 9 & 6 & 0.323 & 0.570 \\
\hline No & 44 & 21 & & \\
\hline \multicolumn{5}{|l|}{ Site } \\
\hline Buccal mucosa & 11 & 0 & 10.758 & 0.056 \\
\hline Floor of mouth & 5 & 4 & & \\
\hline Gingiva & 5 & 4 & & \\
\hline Oropharynx and soft palate & 2 & 2 & & \\
\hline Hard palate & 3 & 1 & & \\
\hline Tongue & 27 & 16 & & \\
\hline
\end{tabular}

survival (OS, $p=0.114$ ) and disease-free survival (DFS, $p=$ 0.044) (Figures 2C,D).

\section{p16 Expression and Its Correlations With Clinicopathology in OSCC Cohort}

To separate the HPV-related cases in OSCC, p16 immunohistochemistry was utilized in the cohort. The expression of p16 was observed in $33.75 \%$ of the primary OSCC tumors (27/80), distinguished by strong staining in $>70 \%$ (scored 4) of tumor cells. p16 was homogeneously detected in the nuclei and cytoplasm of tumor cells (Figure 3A).

The relationship between the p16 expression and clinicopathological factors was shown in Table 2 . The expression of p16 was strongly associated with earlier TNM classification by WHO $(p=0.046)$ and lymph node metastasis $(p=0.003)$, but not with tumor site, age, gender, tumor size, pathology differentiation, cigarette and drinking history.

The relationship between the p16 expression level and patient overall survival was further assessed by Kaplan-Meier survival analysis. The overall survival rate was markedly down-regulated in the p16-negative group compared with the p16-positive group.
The Kaplan-Meier curves indicated that patients with p16positive staining exhibited better OS $(p=0.015)$ and DFS $(p=0.008)$ (Figures 3B,C).

\section{Tomm34 Expression in HPV-Positive OSCC Group and HPV-Negative OSCC Group}

HPV-related status of this OSCC cohort has been described by p16 staining. To further analyze the relationship of Tomm34 and HPV status, the expression of Tomm34 was reassessed in HPV-positive OSCC $(n=27)$ and HPV-negative OSCC $(n=53)$, respectively. As shown in Table 3, the higher expression of Tomm34 was consistently correlated with larger tumor size ( $p=0.01$ in HPV-positive OSCC and $p=$ 0.004 in HPV-negative OSCC) and higher TNM classification ( $p=0.008$ in HPV-positive OSCC and $p<0.001$ in HPVnegative OSCC). However, drinking history was only related with Tomm34-postive expression group in HPV-positive OSCC $(p=0.044)$, while positive node category was only related with Tomm34-postive expression in HPV-negative OSCC $(p=0.001)$.

Furthermore, survival outcomes of four subgroups based on the expression of Tomm34 and p16 were examined (Figures 4A,B). Detailed pairwise comparisons of groups were shown in Table 4 (OS) and Table 5 (DFS). In HPV-positive OSCC subgroups, the survival curves of Tomm34 (+) p16 (+) group and Tomm34 (-) p16 (+) group exhibited rather little difference (OS, $p=0.824$; DFS, $p=0.782$ ). While in HPV-negative OSCC, statistically decreased OS $(p=0.046)$ and DFS $(p=0.020)$ rates were observed between Tomm34 (+) p16 (-) group and Tomm34 (-) p16 (-) group. In addition, Tomm34 (+) p16 $(-)$ group also showed dramatically decreased survival rates compared with Tomm34 (+) p16 (+) group (OS, $p=0.014$; DFS, $p=0.008)$ and Tomm34 (-) p16 (+) group (OS, $p=0.050$; DFS, $p=0.015)$.

\section{DISCUSSION}

Mitochondria plays an important role in cell energy metabolism, senescence, apoptosis, and free radical formation [23]. Translocase of Outer Mitochondrial Membrane (TOMM) is one of the important proteins to maintain the normal function of mitochondria [24]. Tomm34, as a member of the outer mitochondrial membrane proteins, is evenly distributed between the cytoplasm and the outer mitochondrial membrane, and interacts with mitochondrial proteins in the cytoplasm to maintain the pre-protein in an unfolded and easy-to-introd state, and performs the function of a transport protein $[10,11]$. Due to the instability of tumor genome and the lack of essential nutrients and oxygen, tumor cells need a lot of carbohydrates, proteins and other substances for growth and reproduction [25]. Tomm34 is considered to be increased as a component of compensatory adaptations to maintain normal rates of protein import in response to mitochondrial abnormalities in tumor. Our immunostaining data showed that Tomm34 stained predominantly and homogeneously in 
TABLE 3 | Association between IHC expression of Tomm34 and clinicopathological parameters separated by p16-staining status ( $\mathrm{n}=80$ ).

\begin{tabular}{|c|c|c|c|c|c|c|c|c|}
\hline & \multicolumn{4}{|c|}{ p16-positive OSCC $(n=27)$} & \multicolumn{4}{|c|}{ p16-negative OSCC $(n=53)$} \\
\hline & \multicolumn{2}{|c|}{ Tomm34 expression } & \multicolumn{2}{|c|}{ Significance } & \multicolumn{2}{|c|}{ Tomm34 expression } & \multicolumn{2}{|c|}{ Significance } \\
\hline & Negative & Positive & $x^{2}$ & $\begin{array}{c}p \\
\text { Value }\end{array}$ & Negative & Positive & $x^{2}$ & $\begin{array}{c}p \\
\text { Value }\end{array}$ \\
\hline \multicolumn{9}{|l|}{ Age } \\
\hline$\leq 60$ (years) & 10 & 11 & 0.024 & 0.877 & 14 & 22 & 0.064 & 0.801 \\
\hline$>60$ (years) & 2 & 4 & & & 6 & 11 & & \\
\hline \multicolumn{9}{|l|}{ Gender } \\
\hline Male & 8 & 10 & 0.000 & 1.000 & 15 & 22 & 0.410 & 0.522 \\
\hline Female & 4 & 5 & & & 5 & 11 & & \\
\hline \multicolumn{9}{|l|}{ Tumor size } \\
\hline $\mathrm{T} 1 / \mathrm{T} 2$ & 12 & 7 & 6.717 & 0.010 & 18 & 17 & 8.224 & 0.004 \\
\hline T3/T4 & 0 & 8 & & & 2 & 16 & & \\
\hline \multicolumn{9}{|l|}{ Node category } \\
\hline $\mathrm{N}^{-}$ & 11 & 12 & 0.902 & 0.762 & 16 & 11 & 10.852 & 0.001 \\
\hline $\mathrm{N}^{+}$ & 1 & 3 & & & 4 & 22 & & \\
\hline \multicolumn{9}{|l|}{ Pathology differentiation } \\
\hline Well & 6 & 5 & 0.232 & 0.630 & 8 & 16 & 0.362 & 0.547 \\
\hline Moderate/poor & 6 & 10 & & & 12 & 17 & & \\
\hline \multicolumn{9}{|l|}{ TNM classification } \\
\hline$|/| \mid$ & 11 & 5 & 6.497 & 0.008 & 14 & 5 & 16.290 & $<0.001$ \\
\hline III/IV & 1 & 10 & & & 6 & 28 & & \\
\hline \multicolumn{9}{|l|}{ Smoking } \\
\hline Yes & 4 & 8 & 1.080 & 0.299 & 4 & 10 & 0.680 & 0.410 \\
\hline No & 8 & 7 & & & 16 & 23 & & \\
\hline \multicolumn{9}{|l|}{ Alcohol } \\
\hline Yes & 0 & 6 & 4.074 & 0.044 & 2 & 7 & 0.458 & 0.499 \\
\hline No & 12 & 9 & & & 18 & 26 & & \\
\hline \multicolumn{9}{|l|}{ Site } \\
\hline Buccal mucosa & 0 & 0 & 4.712 & 0.403 & 2 & 9 & 4.150 & 0.576 \\
\hline Floor of mouth & 1 & 3 & & & 2 & 3 & & \\
\hline Gingiva & 1 & 3 & & & 2 & 3 & & \\
\hline Oropharynx and soft palate & 2 & 0 & & & 0 & 2 & & \\
\hline Hard palate & 0 & 1 & & & 1 & 2 & & \\
\hline Tongue & 8 & 8 & & & 13 & 14 & & \\
\hline
\end{tabular}
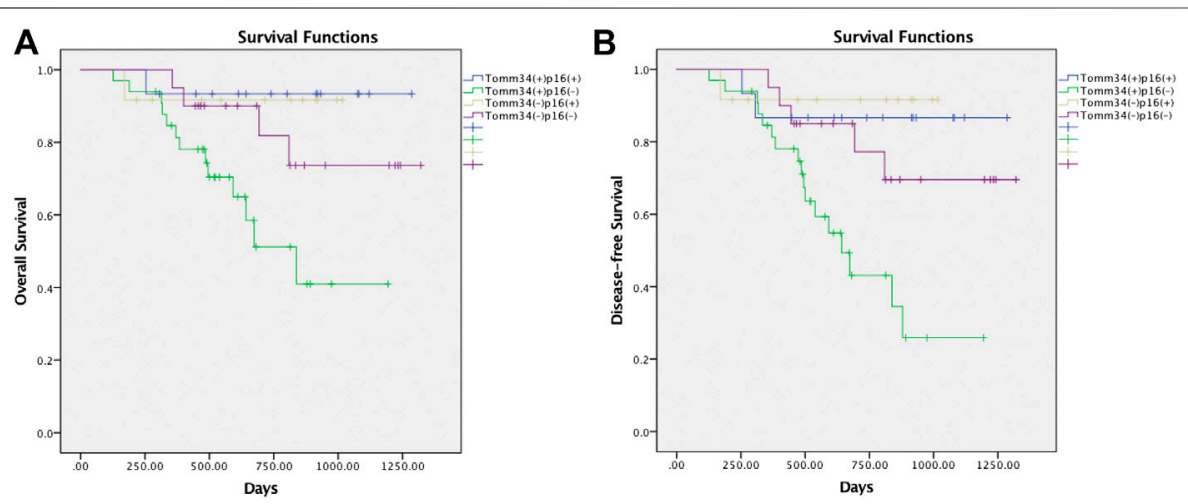

FIGURE 4 | (A) Survival curves of overall survival (OS) in OSCC cohort determined by Tomm34 and p16 expression. (B) Survival curves of disease-free survival (DFS) in OSCC cohort determined by Tomm34 and p16 expression.

the cytoplasm rather than dot cluster coloring in mitochondria, suggesting it was involved in the transport of mitochondrial preproteins in an unfolded state prior to import, which was in agreement with others report.
Tomm34, a co-chaperone of Hsp70 and Hsp90, contains two TPR domains, N-terminal TPR1 and C-terminal TPR2 which binds to the highly conserved EEVD-COOH motif present in the C-terminal domain of Hsp70/Hsp90 [13, 14, 
TABLE 4 | Pairwise comparisons of Overall Survival Rates.

\begin{tabular}{|c|c|c|c|c|c|c|c|c|}
\hline & \multicolumn{2}{|c|}{ Tomm34 (+), p16 (+) } & \multicolumn{2}{|c|}{ Tomm34 (+), p16 (-) } & \multicolumn{2}{|c|}{ Tomm34 (-), p16 (+) } & \multicolumn{2}{|c|}{ Tomm34 (-), p16 (-) } \\
\hline & Chi-square & Sig & Chi-square & Sig & Chi-square & Sig & Chi-square & Sig \\
\hline Tomm34 (+),p16 (+) & & & 6.007 & 0.014 & 0.050 & 0.824 & 0.979 & 0.322 \\
\hline Tomm34 (+), p16 (-) & 6.007 & 0.014 & & & 3.835 & 0.050 & 3.980 & 0.046 \\
\hline Tomm34 (-), p16 (+) & 0.050 & 0.824 & 3.835 & 0.050 & & & 0.522 & 0.470 \\
\hline Tomm34 (-), p16 (-) & 0.979 & 0.322 & 3.980 & 0.046 & 0.522 & 0.470 & & \\
\hline
\end{tabular}

Pairwise comparisons were analyzed by Log Rank (Mantel-Cox).

TABLE 5 | Pairwise comparisons of Disease-free Survival Rates.

\begin{tabular}{|c|c|c|c|c|c|c|c|c|}
\hline & \multicolumn{2}{|c|}{ Tomm34 (+), p16 (+) } & \multicolumn{2}{|c|}{ Tomm34 (+), p16 (-) } & \multicolumn{2}{|c|}{ Tomm34 (-), p16 (+) } & \multicolumn{2}{|c|}{ Tomm34 (-), p16 (-) } \\
\hline & Chi-square & Sig & Chi-square & Sig & Chi-square & Sig & Chi-square & Sig \\
\hline Tomm34 (+),p16 (+) & & & 6.954 & 0.008 & 0.077 & 0.782 & 0.500 & 0.480 \\
\hline Tomm34 (+), p16 (-) & 6.954 & 0.008 & & & 5.964 & 0.015 & 5.447 & 0.020 \\
\hline Tomm34 (-), p16 (+) & 0.077 & 0.782 & 5.964 & 0.015 & & & 0.896 & 0.344 \\
\hline Tomm34 (-), p16 (-) & 0.500 & 0.480 & 5.447 & 0.020 & 0.896 & 0.344 & & \\
\hline
\end{tabular}

Pairwise comparisons were analyzed by Log Rank (Mantel-Cox).

26, 27]. As increased chaperone activities are a universal feature of cancer [28], Hsp70-Tomm34-Hsp90 has been confirmed to be upregulated in a variety of cancers, including liver cancer [16], lung cancer [29], gastric cancer [30] and breast cancer [31], etc. Several studies have reported the immunoreactivity of Hsp70 and Hsp90 in OSCC, which were corelated with poor prognosis [32-35]. Tumor cells need a lot of carbohydrates, proteins and other substances for growth and proliferation which may up-regulate Hsp70 and Hsp90 proteins to synthesize more proteins [36]. Tomm34, as their co-chaperone, may also promote tumor growth by affecting the folding of Hsp70 and Hsp90. In the studied OSCC cohort, $33.75 \%$ of the primary tumors were p16 positive $(27 / 80)$, which was in accordance with previous reports $(10.0-38.1 \%)[5,9]$. Furthermore, our data showed that the expression of Tomm34 was observed in $60 \%$ of the primary OSCC (48/80). The higher expression level of Tomm34 was closely related with higher TNM classification of OSCC $(p<0.001)$ and larger tumor size $(p<0.001)$ in different HPV status. Lymph node metastasis $(p=0.001)$ was related with HPV-negative OSCC, while drinking history was related with HPV-positive OSCC $(p=0.044)$. Most notably, we have found that Tomm34 was associated with poor survival in $\mathrm{HPV}$-negative OSCC rather than HPV-positive OSCC. These data are comparable to the findings that Tomm34 is a marker of poor outcome in bladder cancer and a predictor of distant metastasis in breast cancer.

Meanwhile, our data indicates that Tomm34 is a more sensitive prognosis marker for HPV-negative OSCC patients which is useful to evaluate patience's risk of death. The mechanism for high level expression of Tomm34 is still unclear from our studies. As we known, TP53 mutation is a famous carcinogenetic factor of OSCC, which leads to the instability of the tumor genome [37]. Tomm34 expression levels indicates poor prognosis for p53-mutant epithelial ovarian cancer [20]. Our bioinformatic analysis indicated that higher expression of Tomm34 was detected in TP53-mutant OSCC compared with TP53-nonmutant OSCC and normal tissue $(p<$ 0.001) (Supplementary Figure S1), and further research will be required to elucidate the regulation mechanisms. Recently, researchers have found Tomm34 is transcriptionally regulated by NRF-1 and NRF-2 under cancer stress and hyperactive condition during metabolic reprogramming $[38,39]$. It has been shown that mice without Tomm 34 expression can grow and reproduce normally, which has indicated that Tomm34 is not necessary for normal growth and development [40]. Tomm34 is not expressed in most important organs such as heart, liver, kidney and lung, which indicates that the side effects of using Tomm34 as an anti-cancer target may be minimal. Thus, clinical studies of vaccine therapy with an artificially synthesized cancer peptide based on the amino acid sequence of Tomm34 are ongoing. A phase I clinical trial of a peptide vaccine ring finger protein 43 (RNF43) and Tomm34 combined with uracil-tegafur (UFT)/LV for patients with metastatic colorectal cancer has been done in Japan, including the safety and immunological responsiveness of this combination therapy [41]. Our data has provided evidence that Tomm34 is closely associated with clinicopathological parameters and may serve as a useful prognostic indicator and potential therapeutic target in OSCC, especially in HPV-negative OSCC.

\section{CONCLUSION}

In summary, our study provides evidence that co-chaperone Tomm34 is frequently expressed in OSCC and may be a useful factor to provide prognostic information. Higher level of Tomm34 correlates with poorer overall survival and diseasefree survival in HPV-negative OSCC but not in HPV-positive OSCC. Tomm34 may serve as a potential tumor biomarker for OSCC patients, especially in HPV-negative OSCC. 


\section{DATA AVAILABILITY STATEMENT}

The raw data supporting the conclusions of this article will be made available by the authors, without undue reservation.

\section{ETHICS STATEMENT}

The study protocol was approved by the Clinical Research Ethics Committee of Hospital of Stomatology, Guanghua School of Stomatology, Sun Yat-sen University. The patients/participants provided their written informed consent to participate in this study.

\section{AUTHOR CONTRIBUTIONS}

Conceived and designed the experiments: MC, MX. Performed the experiments: MC, RT, YH. Analyzed the data: MC, XC. Bioinformatic analysis: QK, KG. Contributed reagents/ materials/analysis tools: MC, MX. Wrote the paper: MC, MX. $\mathrm{MC}, \mathrm{RT}, \mathrm{YH}$ contribute equally to this work.

\section{REFERENCES}

1. Rahman QB, Iocca O, Kufta K, Shanti RM. Global burden of head and neck cancer. Oral Maxillofac Surg Clin North Am (2020) 32(3):367-75. doi:10.1016/j. coms.2020.04.002

2. Mourad M, Jetmore T, Jategaonkar AA, Moubayed S, Moshier E, Urken ML. Epidemiological trends of head and neck cancer in the United States: a seer population study. J Oral Maxillofac Surg (2017) 75(12):2562-72. doi:10.1016/j. joms.2017.05.008

3. Ding D, Stokes W, Eguchi M, Hararah M, Sumner W, Amini A, et al. Association between lymph node ratio and recurrence and survival outcomes in patients with oral cavity cancer. JAMA Otolaryngol Head Neck Surg (2019) 145(1):53-61. doi:10.1001/jamaoto.2018.2974

4. Dionne KR, Warnakulasuriya S, Zain RB, Cheong SC. Potentially malignant disorders of the oral cavity: current practice and future directions in the clinic and laboratory. Int J Cancer (2015) 136(3):503-15. doi:10.1002/ijc.28754

5. Zaravinos A. An updated overview of HPV-associated head and neck carcinomas. Oncotarget (2014) 5(12):3956-69. doi:10.18632/oncotarget.1934

6. Huang SH, O'Sullivan B. Overview of the 8th edition TNM classification for head and neck cancer. Curr Treat Options Oncol (2017) 18(7):13. doi:10.1007/ s11864-017-0484-y

7. Mueller S. Update from the 4th edition of the world health organization of head and neck tumours: tumours of the oral cavity and mobile tongue. Head Neck Pathol (2017) 11(1):33-40. doi:10.1007/s12105-017-0792-3

8. Westra WH, Lewis JS, Jr. Update from the 4th edition of the world health organization classification of head and neck tumours: oropharynx. Head Neck Pathol (2017) 11(1):41-7. doi:10.1007/s12105-017-0793-2

9. Paver EC, Currie AM, Gupta R, Dahlstrom JE. Human papilloma virus related squamous cell carcinomas of the head and neck: diagnosis, clinical implications and detection of HPV. Pathology (2020) 52(2):179-91. doi:10.1016/j.pathol. 2019.10.008

10. Nuttall SD, Hanson BJ, Mori M, Hoogenraad NJ. hTom34: a novel translocase for the import of proteins into human mitochondria. DNA Cell Biol (1997) 16(9):1067-74. doi:10.1089/dna.1997.16.1067

11. Yang CS, Weiner H. Yeast two-hybrid screening identifies binding partners of human Tom 34 that have ATPase activity and form a complex with Tom34 in the cytosol. Arch Biochem Biophys (2002) 400(1):105-10. doi:10.1006/abbi. 2002.2778

\section{FUNDING}

Supported by Foundation and Applied Basic Research Fund of Guangdong (Grand No. 2020A1515010529; National Natural Science Foundation of China (Grand No. 81602384); Special Funds for Scientific and Technological Innovation and Cultivation of Guangdong University Students (Grand No. pdjh2021a0002).

\section{CONFLICT OF INTEREST}

The authors declare that the research was conducted in the absence of any commercial or financial relationships that could be construed as a potential conflict of interest.

\section{SUPPLEMENTARY MATERIAL}

The Supplementary Material for this article can be found online at: https://www.por-journal.com/articles/10.3389/pore.2021.641042/ full\#supplementary-material.

12. Chewawiwat N, Yano M, Terada K, Hoogenraad NJ, Mori M. Characterization of the novel mitochondrial protein import component, Tom34, in mammalian cells. J Biochem (1999) 125(4):721-7. doi:10.1093/oxfordjournals.jbchem. a022342

13. Faou P, Hoogenraad NJ. Tom34: a cytosolic cochaperone of the Hsp90/Hsp70 protein complex involved in mitochondrial protein import. Biochim Biophys Acta (2012) 1823(2):348-57. doi:10.1016/j.bbamcr.2011.12.001

14. Trcka F, Durech M, Man P, Hernychova L, Muller P, Vojtesek B. The assembly and intermolecular properties of the Hsp70-Tomm34-Hsp90 molecular chaperone complex. J Biol Chem (2014) 289(14):9887-901. doi:10.1074/jbc. M113.526046

15. Matsushita N, Yamamoto S, Inoue Y, Aruga A, Yamamoto M. RT-qPCR analysis of the tumor antigens TOMM34 and RNF43 in samples extracted from paraffin-embedded specimens of colorectal cancer. Oncol Lett (2017) 14(2):2281-7. doi:10.3892/ol.2017.6412

16. Toraih EA, Alrefai HG, Hussein MH, Helal GM, Khashana MS, Fawzy MS. Overexpression of heat shock protein HSP90AA1 and translocase of the outer mitochondrial membrane TOM34 in HCV-induced hepatocellular carcinoma: a pilot study. Clin Biochem (2019) 63:10-7. doi:10.1016/j.clinbiochem.2018. 12.001

17. Ahmed MAH, Ali MH, Abbas HH, Elatrash GA, Foda AAM. Expression of TOMM34 and its clinicopathological correlations in urothelial carcinoma of the bladder. Pathol Oncol Res (2020) 26(1):411-8. doi:10.1007/s12253-0180524-3

18. Aleskandarany MA, Negm OH, Rakha EA, Ahmed MA, Nolan CC, Ball GR, et al. TOMM34 expression in early invasive breast cancer: a biomarker associated with poor outcome. Breast Cancer Res Treat (2012) 136(2): 419-27. doi:10.1007/s10549-012-2249-4

19. Aleskandarany MA, Soria D, Green AR, Nolan C, Diez-Rodriguez M, Ellis IO, et al. Markers of progression in early-stage invasive breast cancer: a predictive immunohistochemical panel algorithm for distant recurrence risk stratification. Breast Cancer Res Treat (2015) 151(2):325-33. doi:10.1007/ s10549-015-3406-3

20. Muller P, Coates PJ, Nenutil R, Trcka F, Hrstka R, Chovanec J, et al. Tomm34 is commonly expressed in epithelial ovarian cancer and associates with tumour type and high FIGO stage. J Ovarian Res (2019) 12(1):30. doi:10.1186/s13048019-0498-0

21. Shimokawa T, Matsushima S, Tsunoda T, Tahara H, Nakamura Y, Furukawa Y. Identification of TOMM34, which shows elevated expression in the majority 
of human colon cancers, as a novel drug target. Int J Oncol (2006) 29(2):381-6. doi:10.3892/ijo.29.2.381

22. Chandrashekar DS, Bashel B, Balasubramanya SAH, Creighton CJ, PonceRodriguez I, Chakravarthi B, et al. UALCAN: a portal for facilitating tumor subgroup gene expression and survival analyses. Neoplasia (2017) 19(8): 649-58. doi:10.1016/j.neo.2017.05.002

23. Dard L, Blanchard W, Hubert C, Lacombe D, Rossignol R. Mitochondrial functions and rare diseases. Mol Aspects Med (2020) 71:100842. doi:10.1016/j. mam.2019.100842

24. Ryan MT, Wagner R, Pfanner N. The transport machinery for the import of preproteins across the outer mitochondrial membrane. Int J Biochem Cell Biol (2000) 32(1):13-21. doi:10.1016/s1357-2725(99)00114-4

25. Gouirand V, Guillaumond F, Vasseur S. Influence of the tumor microenvironment on cancer cells metabolic reprogramming. Front Oncol (2018) 8:117. doi:10.3389/fonc.2018.00117

26. Tsaytler PA, Krijgsveld J, Goerdayal SS, Rudiger S, Egmond MR. Novel Hsp90 partners discovered using complementary proteomic approaches. Cell Stress Chaperones (2009) 14(6):629-38. doi:10.1007/s12192-009-0115-Z

27. Schlegel T, Mirus O, von Haeseler A, Schleiff E. The tetratricopeptide repeats of receptors involved in protein translocation across membranes. Mol Biol Evol (2007) 24(12):2763-74. doi:10.1093/molbev/msm211

28. Calderwood SK, Khaleque MA, Sawyer DB, Ciocca DR. Heat shock proteins in cancer: chaperones of tumorigenesis. Trends Biochem Sci (2006) 31(3):164-72. doi:10.1016/j.tibs.2006.01.006

29. Rodina A, Vilenchik M, Moulick K, Aguirre J, Kim J, Chiang A, et al. Selective compounds define Hsp90 as a major inhibitor of apoptosis in small-cell lung cancer. Nat Chem Biol (2007) 3(8):498-507. doi:10.1038/nchembio.2007.10

30. Giaginis C, Daskalopoulou SS, Vgenopoulou S, Sfiniadakis I, Kouraklis G, Theocharis SE. Heat Shock Protein-27, -60 and -90 expression in gastric cancer: association with clinicopathological variables and patient survival. BMC Gastroenterol (2009) 9:14. doi:10.1186/1471-230X-9-14

31. Pick E, Kluger Y, Giltnane JM, Moeder C, Camp RL, Rimm DL, et al. High HSP90 expression is associated with decreased survival in breast cancer. Cancer Res (2007) 67(7):2932-7. doi:10.1158/0008-5472.CAN-06-4511

32. Priyanka KP, Majumdar S, Kotina S, Uppala D, Balla H. Expression of heat shock protein 70 in oral epithelial dysplasia and oral squamous cell carcinoma: an immunohistochemical study. Contemp Clin Dentistry (2019) 10(2):185-90. doi:10.4103/ccd.ccd_101_18

33. Chanthammachat P, Promwikorn W, Pruegsanusak K, Roytrakul S, Srisomsap C, Chokchaichamnankit D, et al. Comparative proteomic analysis of oral squamous cell carcinoma and adjacent non-tumour tissue from Thailand. Arch Oral Biol (2013) 58(11):1677-85. doi:10.1016/j.archoralbio.2013.08.002

34. Markopoulos AK, Deligianni E, Antoniades DZ. Heat shock protein 70 membrane expression in oral cancer: a possible new target in antineoplastic therapy? Chemotherapy (2009) 55(4):211-4. doi:10. $1159 / 000218099$

35. Lee S-S, Tsai C-H, Ho Y-C, Chang Y-C. The upregulation of heat shock protein 70 expression in areca quid chewing-associated oral squamous cell carcinomas. Oral Oncol (2008) 44(9):884-90. doi:10.1016/j.oraloncology. 2007.11.004

36. Shevtsov M, Balogi Z, Khachatryan W, Gao H, Vigh L, Multhoff G. Membrane-associated heat shock proteins in Oncology: from basic research to new theranostic targets. Cells (2020) 9(5). doi:10.3390/cells9051263

37. Pickering CR, Zhang JX, Yoo SY, Bengtsson L, Moorthy S, Neskey DM, et al. Integrative genomic characterization of oral squamous cell carcinoma identifies frequent somatic drivers. Cancer Discov (2013) 3(7):770-81. doi:10.1158/2159-8290.Cd-12-0537

38. Blesa JR, Prieto-Ruiz JA, Abraham BA, Harrison BL, Hegde AA, HernandezYago J. NRF-1 is the major transcription factor regulating the expression of the human TOMM34 gene. Biochem Cel Biol (2008) 86(1):46-56. doi:10.1139/ o07-151

39. Hayes JD, Dinkova-Kostova AT. The Nrf2 regulatory network provides an interface between redox and intermediary metabolism. Trends Biochem Sci (2014) 39(4):199-218. doi:10.1016/j.tibs.2014.02.002

40. Terada K, Ueno S, Yomogida K, Imai T, Kiyonari H, Takeda N, et al. Expression of Tom 34 splicing isoforms in mouse testis and knockout of Tom34 in mice. J Biochem (2003) 133(5):625-31. doi:10.1093/jb/ mvg080

41. Okuno K, Sugiura F, Hida JI, Tokoro T, Ishimaru E, Sukegawa Y, et al. Phase I clinical trial of a novel peptide vaccine in combination with UFT/LV for metastatic colorectal cancer. Exp Ther Med (2011) 2(1):73-9. doi:10.3892/etm. 2010.182

Copyright $\odot 2021$ Cai, Tan, Huang, Chen, Kong, Guo and Xu. This is an open-access article distributed under the terms of the Creative Commons Attribution License (CC $B Y$ ). The use, distribution or reproduction in other forums is permitted, provided the original author(s) and the copyright owner(s) are credited and that the original publication in this journal is cited, in accordance with accepted academic practice. No use, distribution or reproduction is permitted which does not comply with these terms. 